\title{
Caucanolides A-F, Unusual Antiplasmodial Constituents from a Colombian Collection of the Gorgonian Coral Pseudopterogorgia bipinnata
}

\author{
Claudia A. Ospina, Abimael D. Rodríguez, Juan A. Sánchez, Eduardo Ortega-Barria, Todd L. \\ Capson, and Alejandro M. S. Mayer
}

\section{List of Supporting Information}

Contents

Pages

1. Known Compounds Isolated from Pseudopterogorgia bipinnata S2 During this Investigation

2. ${ }^{1} \mathrm{H}$ NMR $(500 \mathrm{MHz})$ and ${ }^{13} \mathrm{C}$ NMR $(125 \mathrm{MHz})$ Spectra $\left(\mathrm{CDCl}_{3}\right)$ S3 of Caucanolide A (1)

3. ${ }^{1} \mathrm{H}$ NMR $(300 \mathrm{MHz})$ and ${ }^{13} \mathrm{C}$ NMR $(75 \mathrm{MHz})$ Spectra $\left(\mathrm{CDCl}_{3}\right)$ S4 of Caucanolide B (2)

4. ${ }^{1} \mathrm{H}$ NMR $(300 \mathrm{MHz})$ and ${ }^{13} \mathrm{C}$ NMR $(75 \mathrm{MHz})$ Spectra $\left(\mathrm{CDCl}_{3}\right)$ S5 of Caucanolide D (4)

5. ${ }^{1} \mathrm{H}$ NMR $(300 \mathrm{MHz})$ and ${ }^{13} \mathrm{C}$ NMR $(75 \mathrm{MHz})$ Spectra $\left(\mathrm{CDCl}_{3}\right)$ S6 of Caucanolide E (5)

6. COSY Spectrum $\left(\mathrm{CDCl}_{3}\right)$ of Caucanolide A (1)

7. HMQC Spectrum $\left(\mathrm{CDCl}_{3}\right)$ of Caucanolide A (1)

S8

8. HMBC Spectrum $\left(\mathrm{CDCl}_{3}\right)$ of Caucanolide A (1)

S9

9. NOESY Spectrum $\left(\mathrm{CDCl}_{3}\right)$ of Caucanolide A (1) S10

10. COSY Spectrum $\left(\mathrm{CDCl}_{3}\right)$ of Caucanolide E (5) S11

11. HMQC Spectrum $\left(\mathrm{CDCl}_{3}\right)$ of Caucanolide E (5) S12

12. HMBC Spectrum $\left(\mathrm{CDCl}_{3}\right)$ of Caucanolide E (5) S13

13. NOESY Spectrum $\left(\mathrm{CDCl}_{3}\right)$ of Caucanolide E (5) S14 
Known Compounds Isolated from Pseudopterogorgia bipinnata During this Investigation

\section{Pseudopteranes}<smiles>C=C(C)[C@@H]1C=C(CC[C@H](C(=C)C)c2cc(C)c([C@@H](OC(C)=O)C(C)=O)o2)C(=O)O1</smiles>

kallolide A acetate<smiles></smiles>

gersemolide<smiles>C=C(C)C(CCC1=C[C@@H]([C@@H](C(=C)C)C(=O)[C@]2(C)O[C@@]2(C)C(=O)[C@H](O)C(C)C)OC1=O)C(=C)C</smiles>

bipinnapterolide A<smiles>C=C(C)C(CCC1=C[C@@H]([C@@H](C(=C)C)C(=O)/C(O)=C(/C)C(=O)[C@@H](O)[C@@H]2C=C(C)C(=O)O2)OC1=O)C(=C)C</smiles>

kallolide C

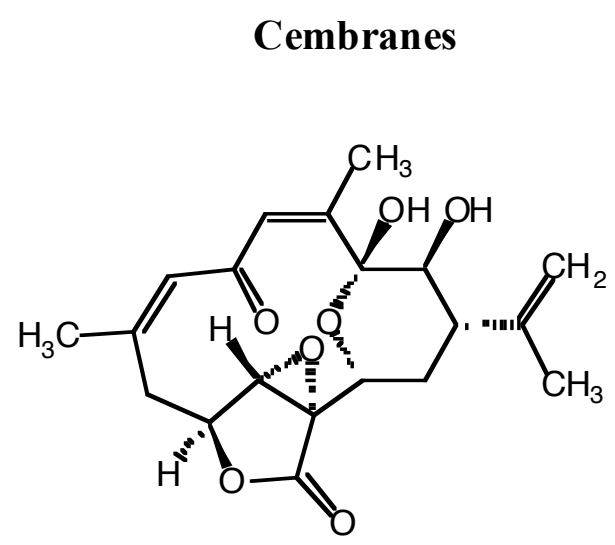

bipinnatolide $\mathrm{F}$

\section{Gersolanes}<smiles>C=C(C)[C@H]1C=C(CC[C@@H](C(C)=O)[C@H](OC(C)=O)C(=O)/C(C)=C\C(=O)C2(C)CC2)C(=O)O1</smiles>

pinnatin $B$ 


\section{${ }^{1} \mathrm{H}$ and ${ }^{13} \mathrm{C}$ NMR Spectra $\left(\mathrm{CDCl}_{3}\right)$ of Caucanolide A (1)}
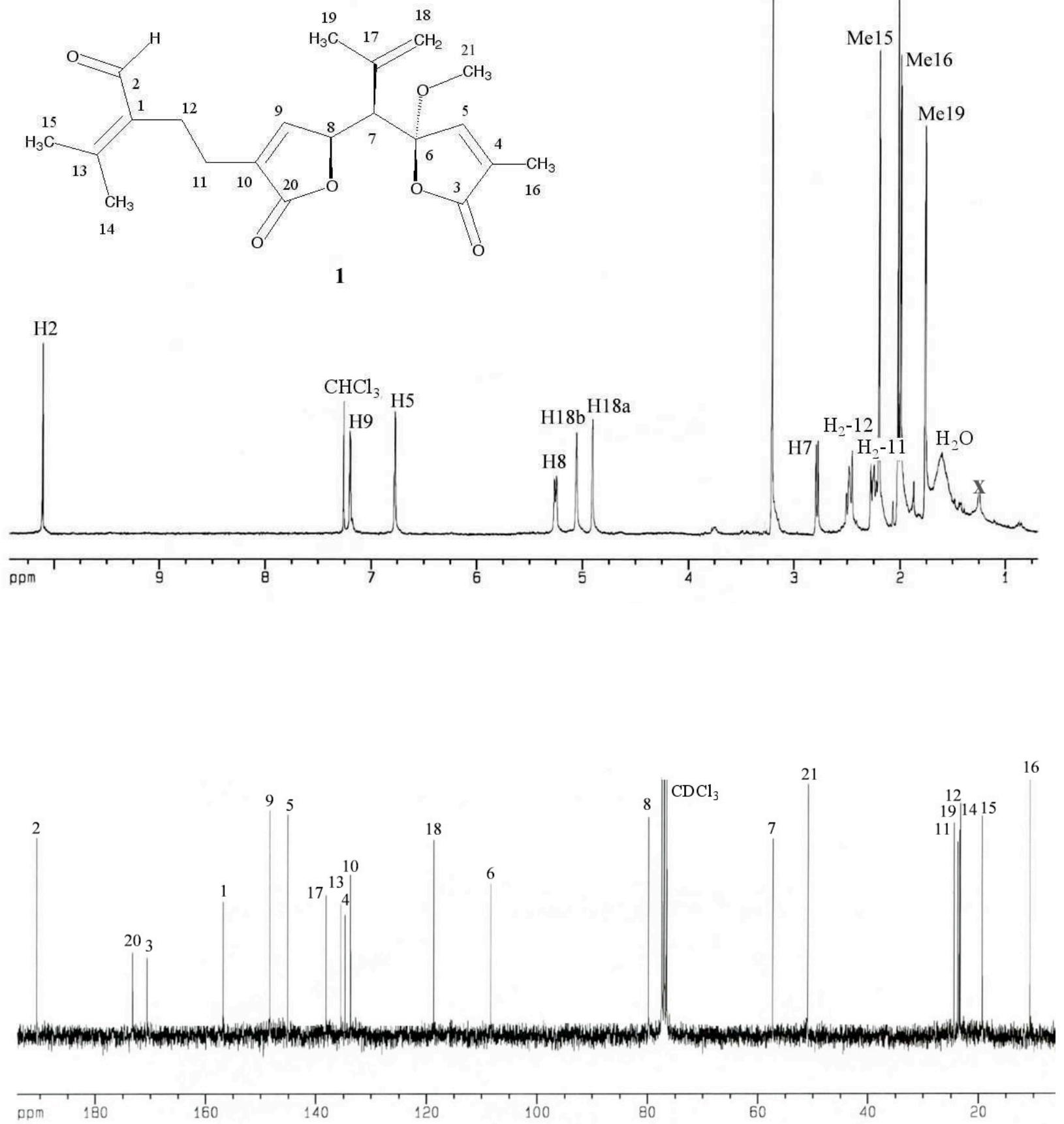


\section{${ }^{1} \mathrm{H}$ and ${ }^{13} \mathrm{C}$ NMR Spectra $\left(\mathrm{CDCl}_{3}\right)$ of Caucanolide B (2)}
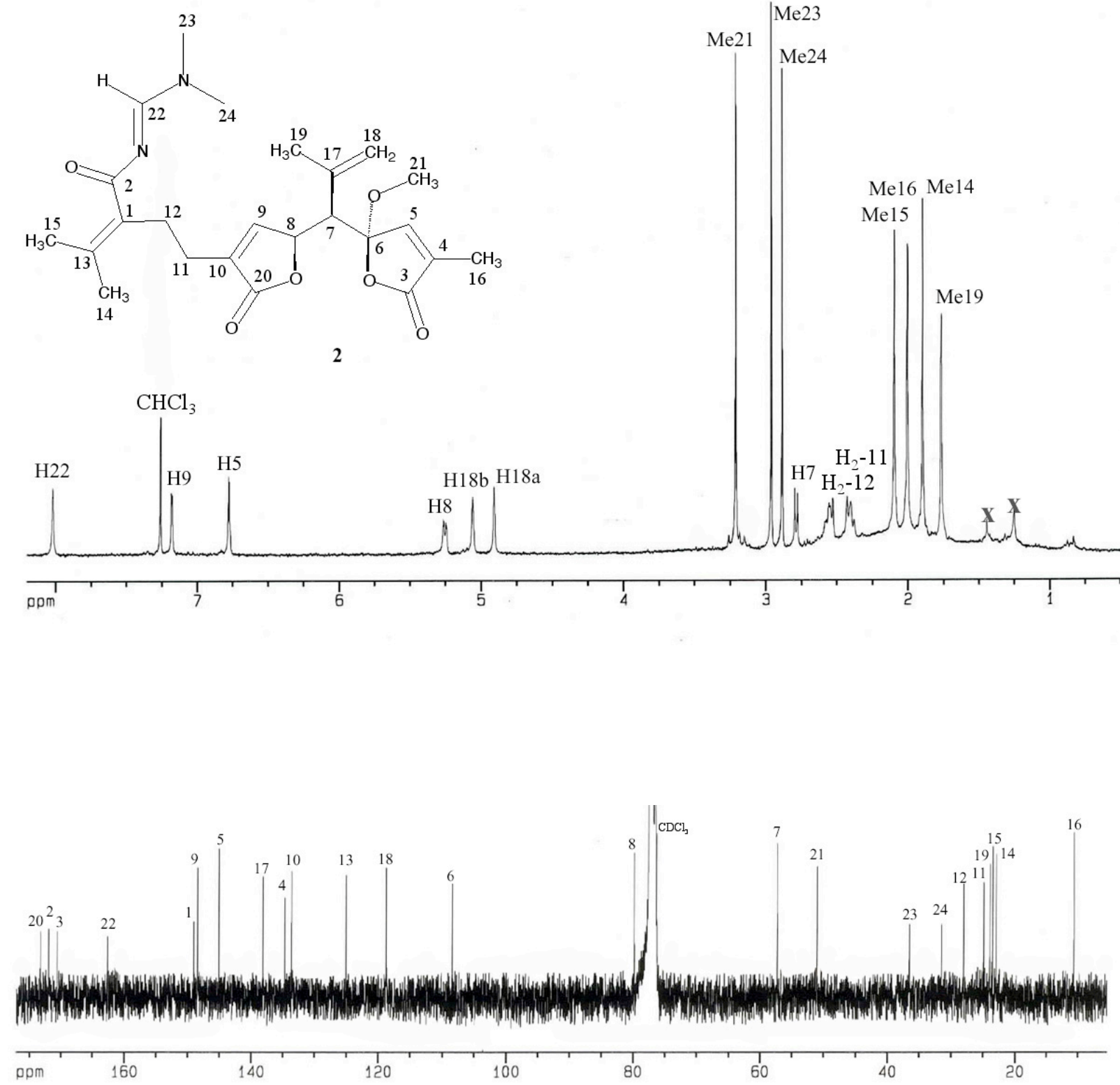
${ }^{1} \mathrm{H}$ and ${ }^{13} \mathrm{C}$ NMR Spectra $\left(\mathrm{CDCl}_{3}\right)$ of Caucanolide D (4)
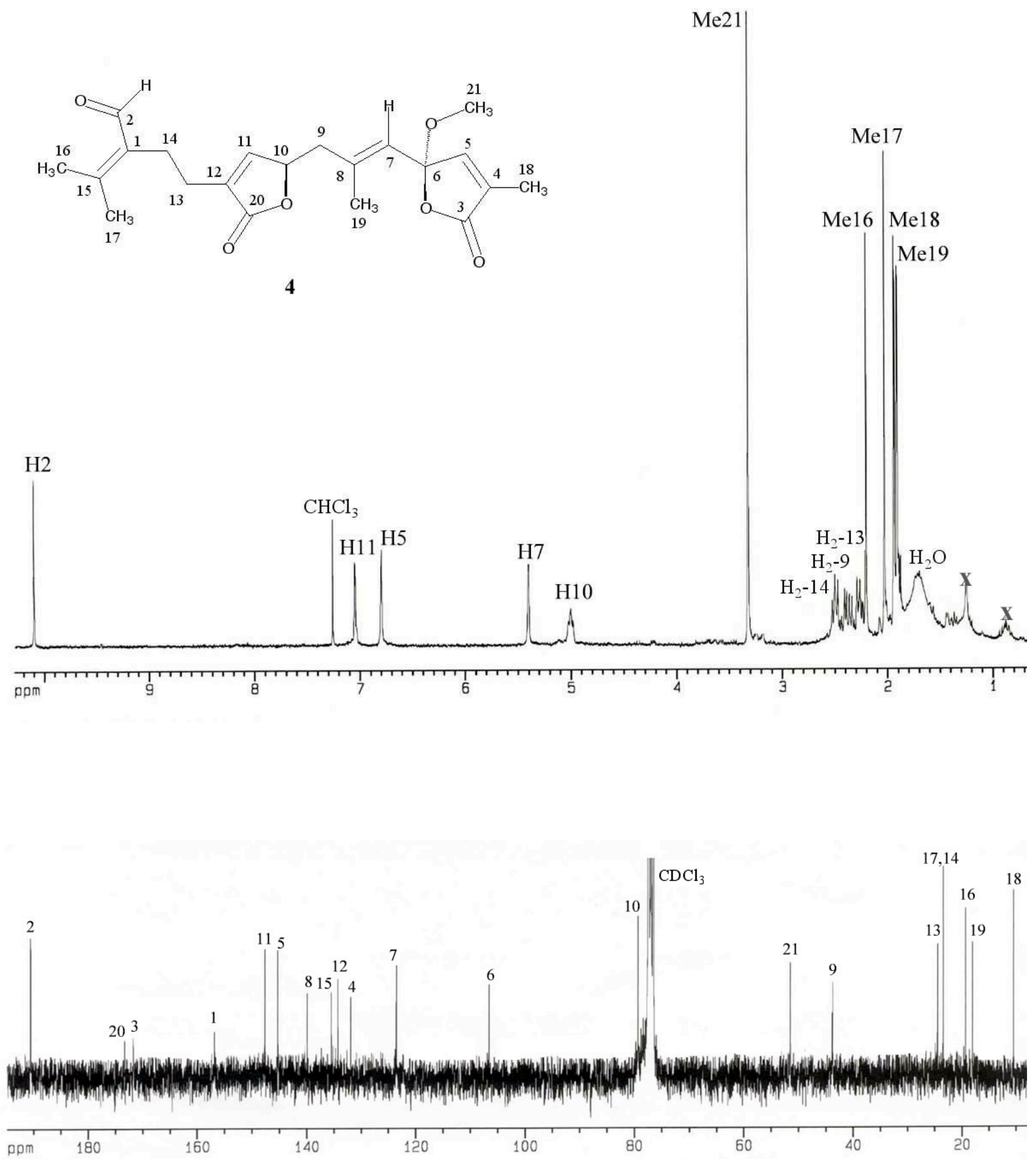
${ }^{1} \mathrm{H}$ and ${ }^{13} \mathrm{C}$ NMR Spectra $\left(\mathrm{CDCl}_{3}\right)$ of Caucanolide E (5)
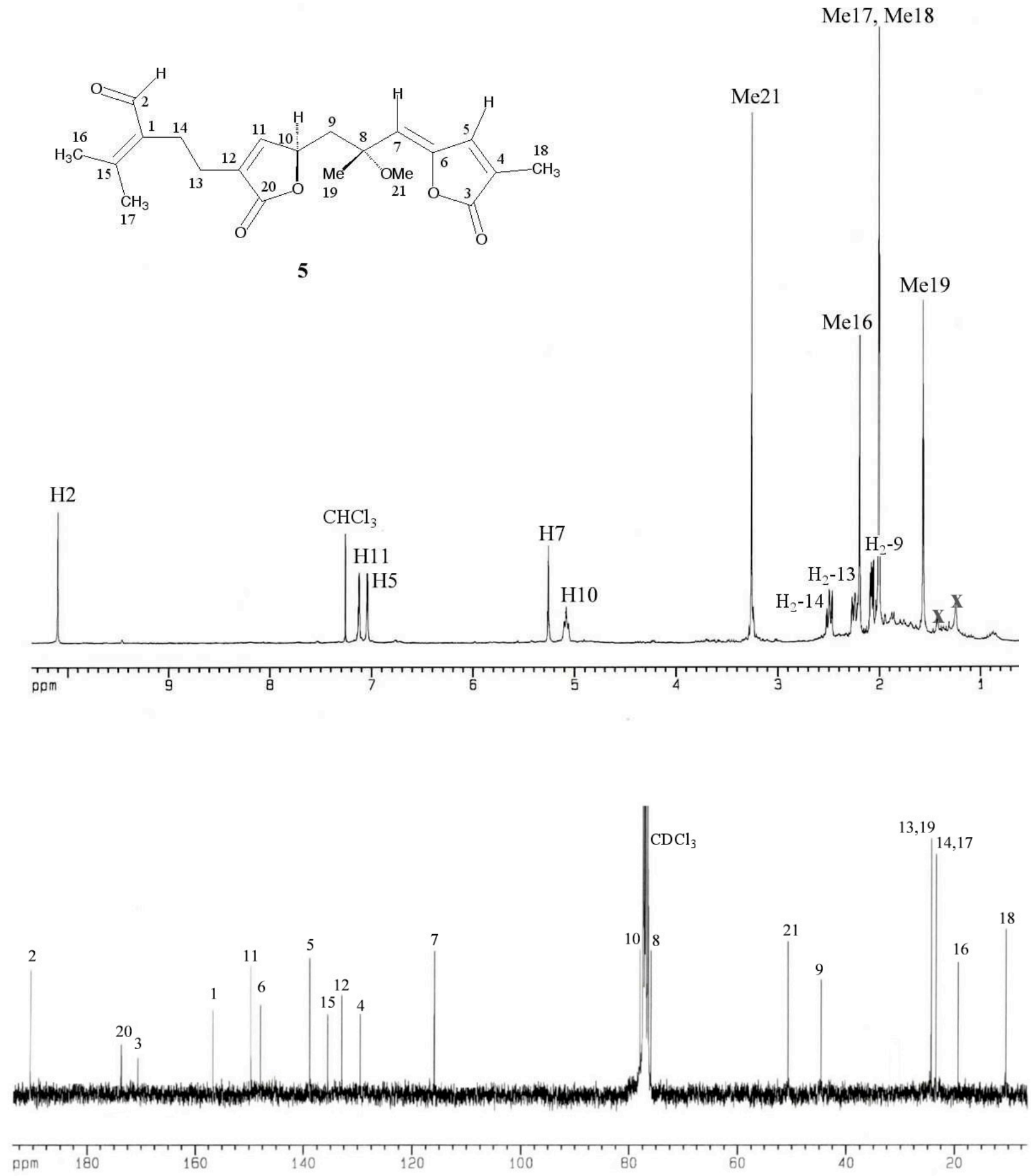
COSY Spectrum $\left(\mathrm{CDCl}_{3}\right)$ of Caucanolide A (1)
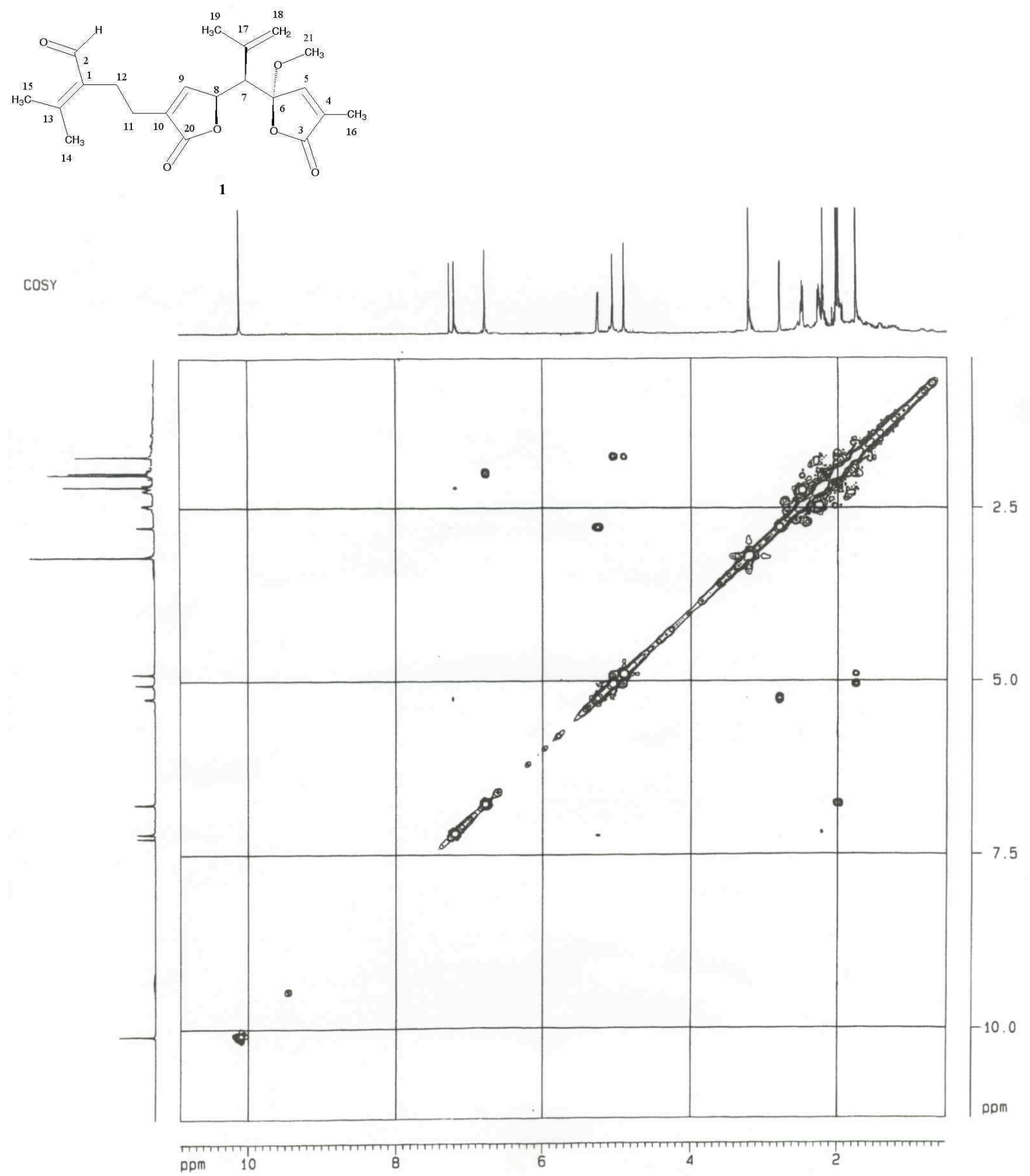


\section{HMQC Spectrum $\left(\mathrm{CDCl}_{3}\right)$ of Caucanolide A (1)}
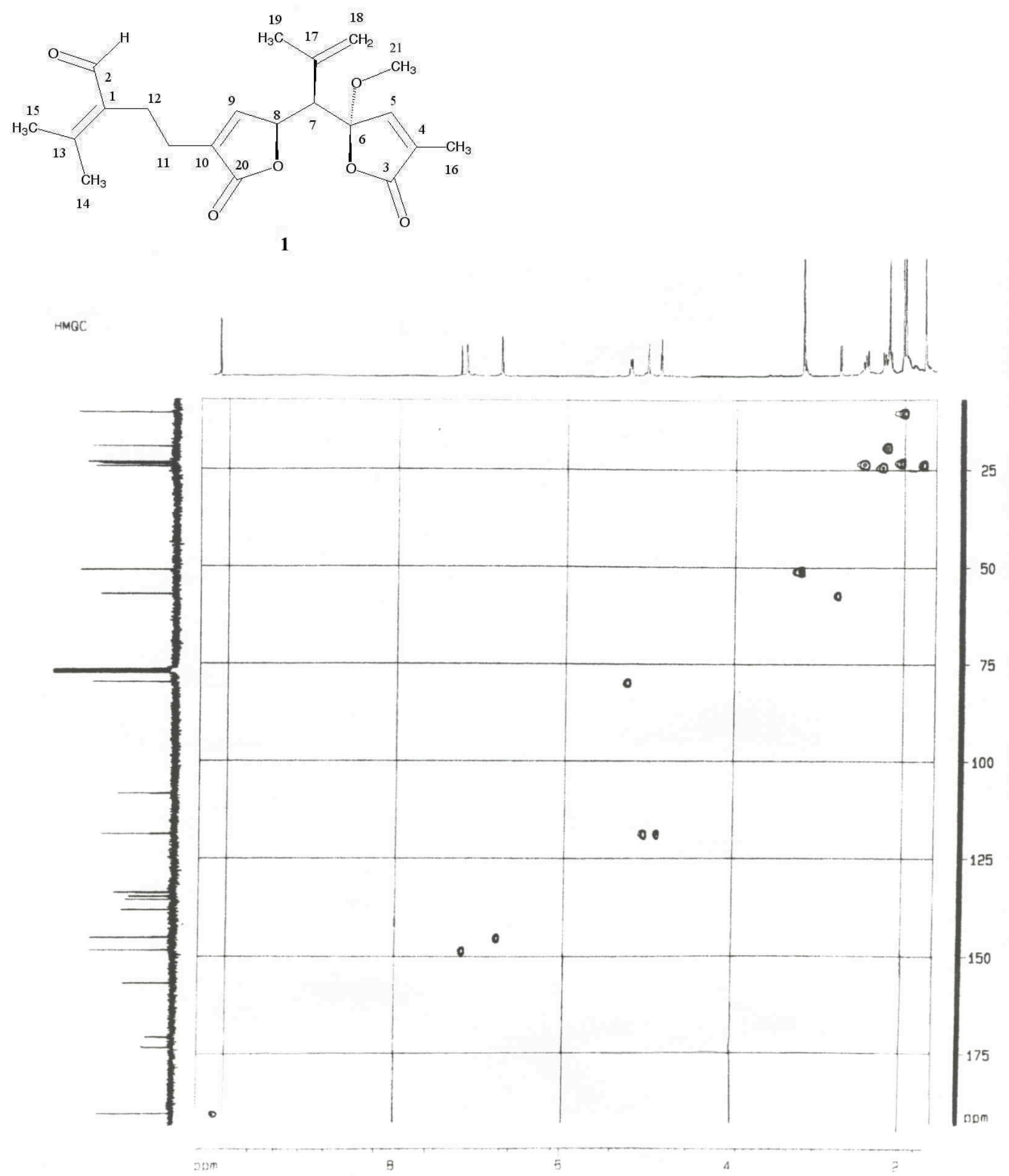


\section{HMBC Spectrum $\left(\mathrm{CDCl}_{3}\right)$ of Caucanolide A (1)}
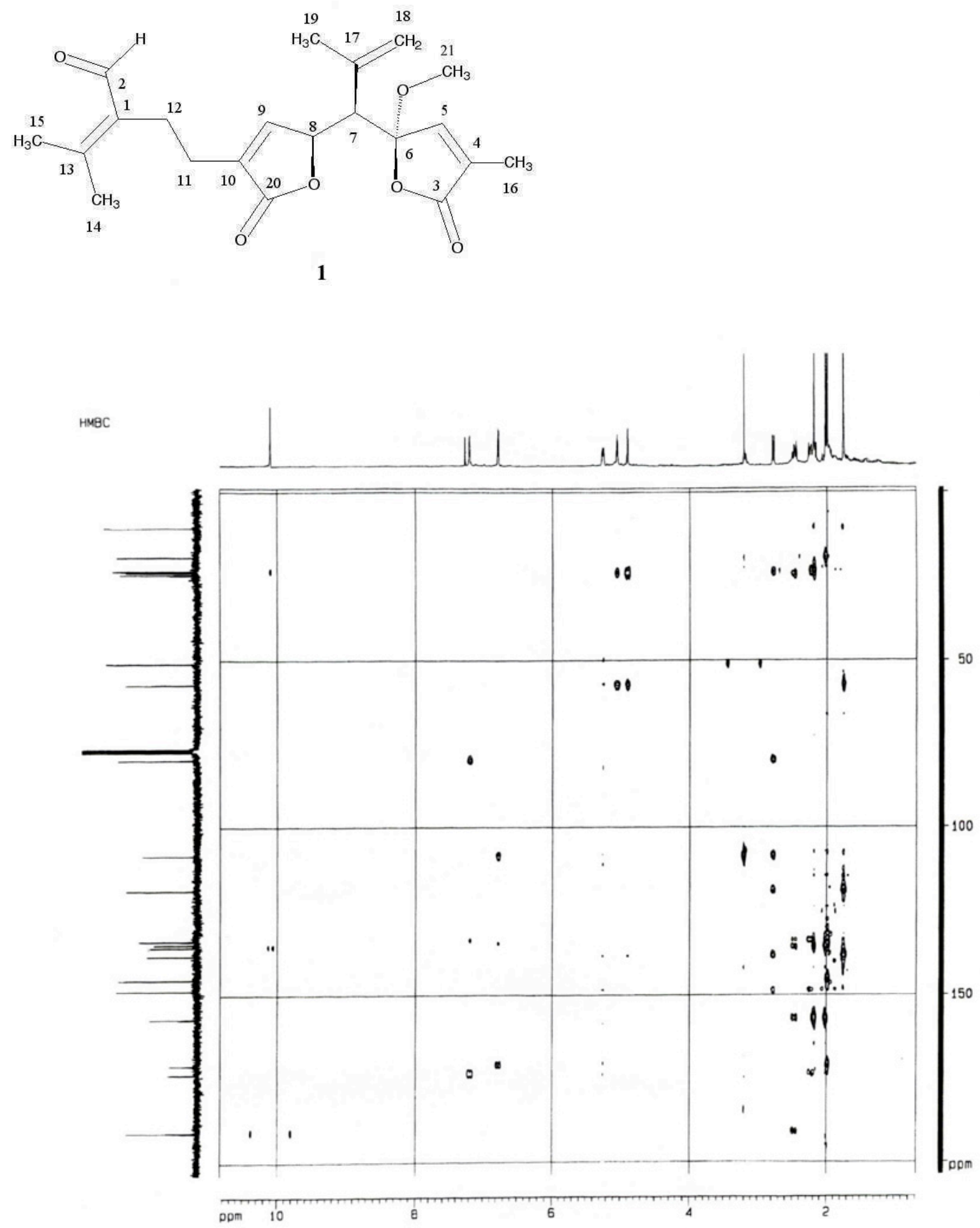


\section{NOESY Spectrum $\left(\mathrm{CDCl}_{3}\right)$ of Caucanolide A (1)}
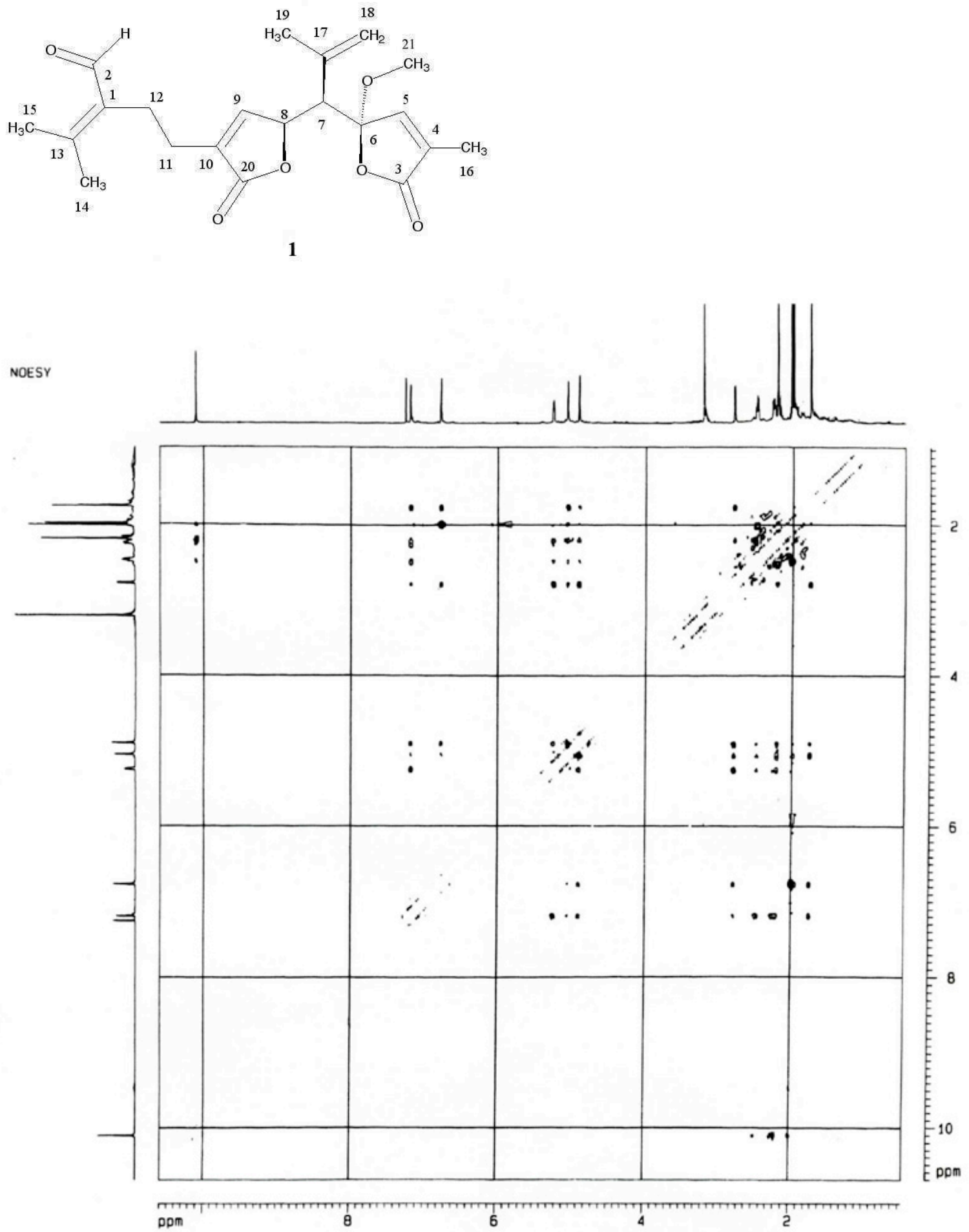
COSY Spectrum $\left(\mathrm{CDCl}_{3}\right)$ of Caucanolide $\mathrm{E}$ (5)

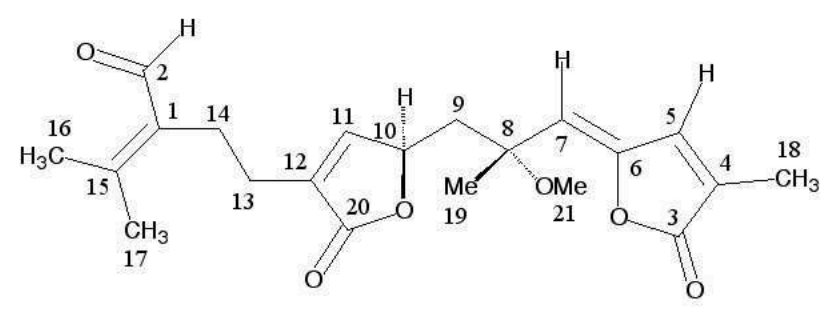

5

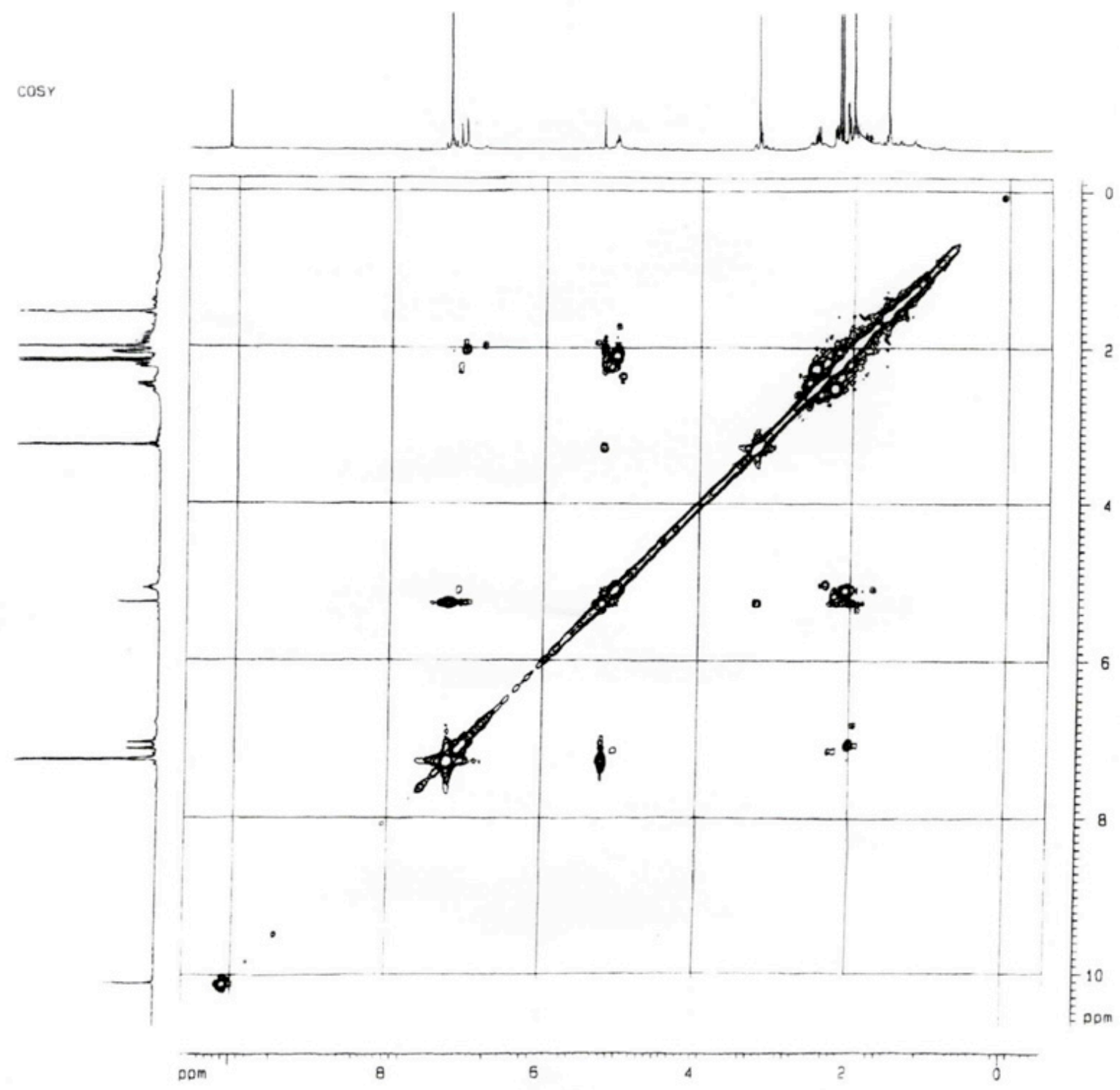




\section{HMQC Spectrum $\left(\mathrm{CDCl}_{3}\right)$ of Caucanolide E (5)}

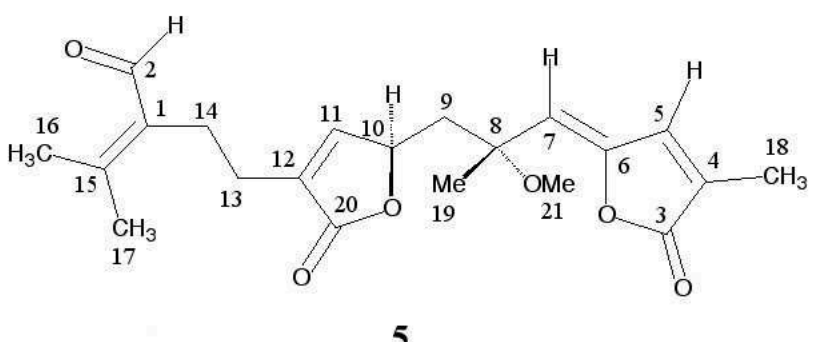

5

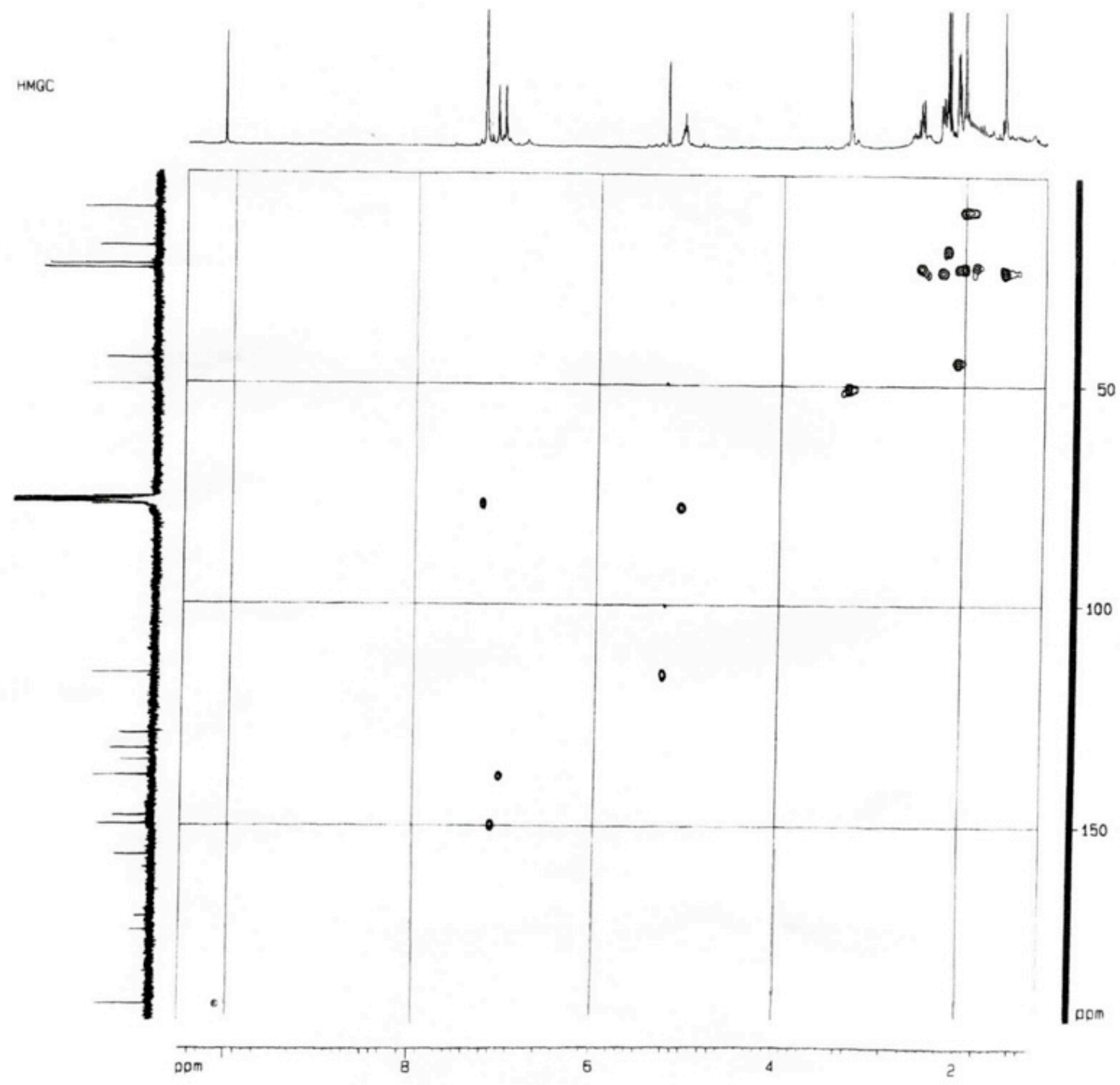


HMBC Spectrum $\left(\mathrm{CDCl}_{3}\right)$ of Caucanolide $\mathrm{E}(5)$

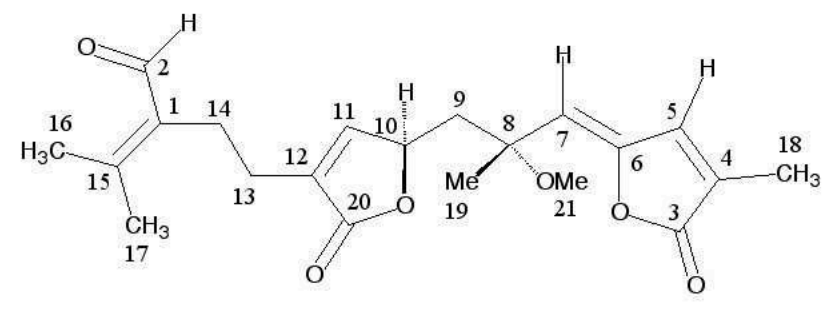

5

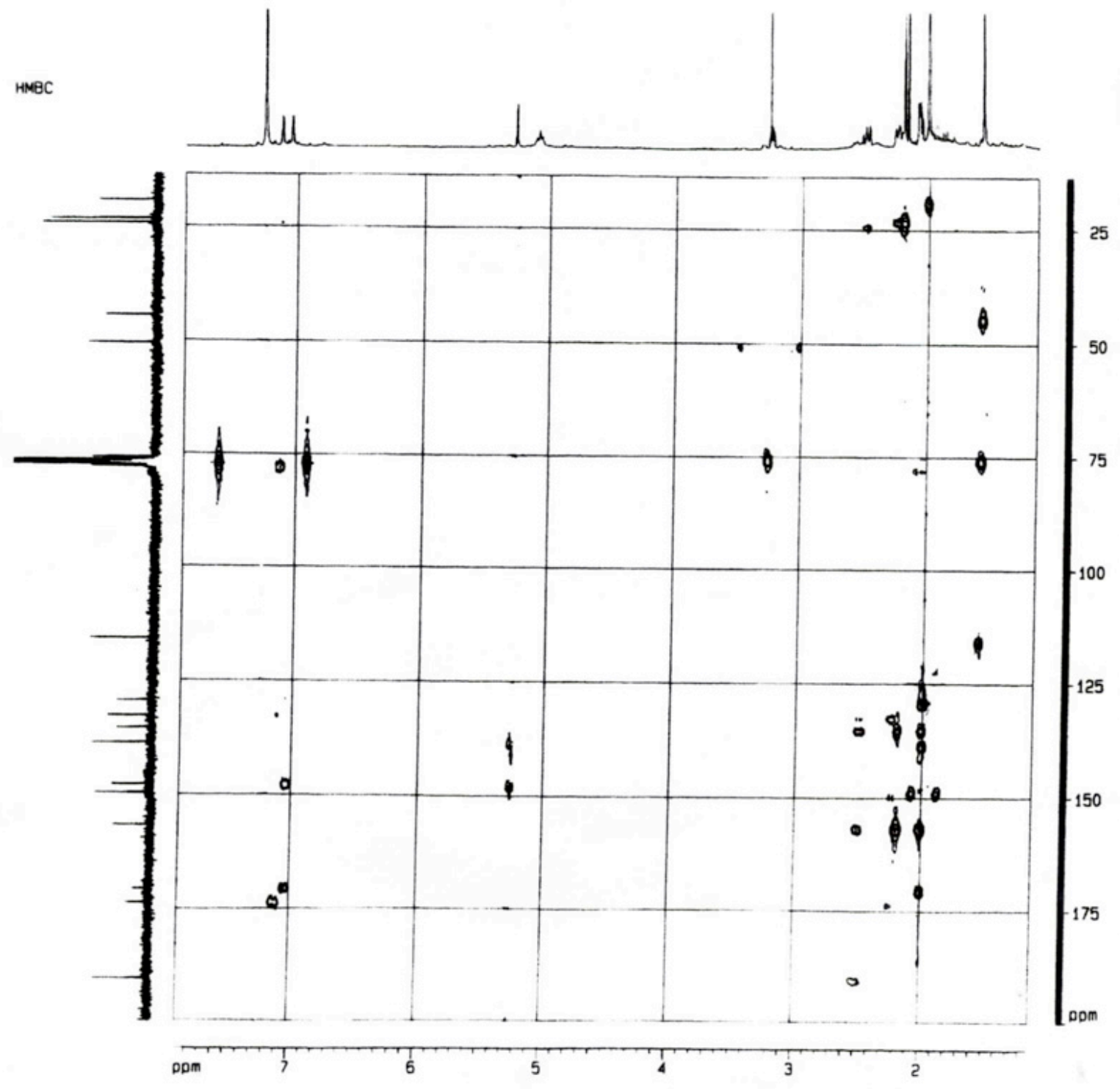


NOESY Spectrum $\left(\mathrm{CDCl}_{3}\right)$ of Caucanolide E (5)

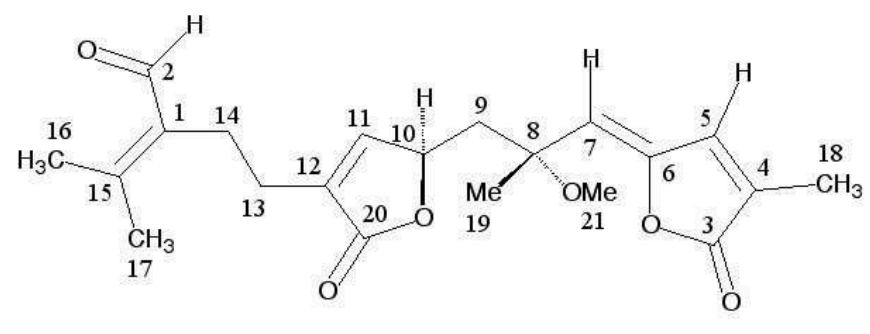

5

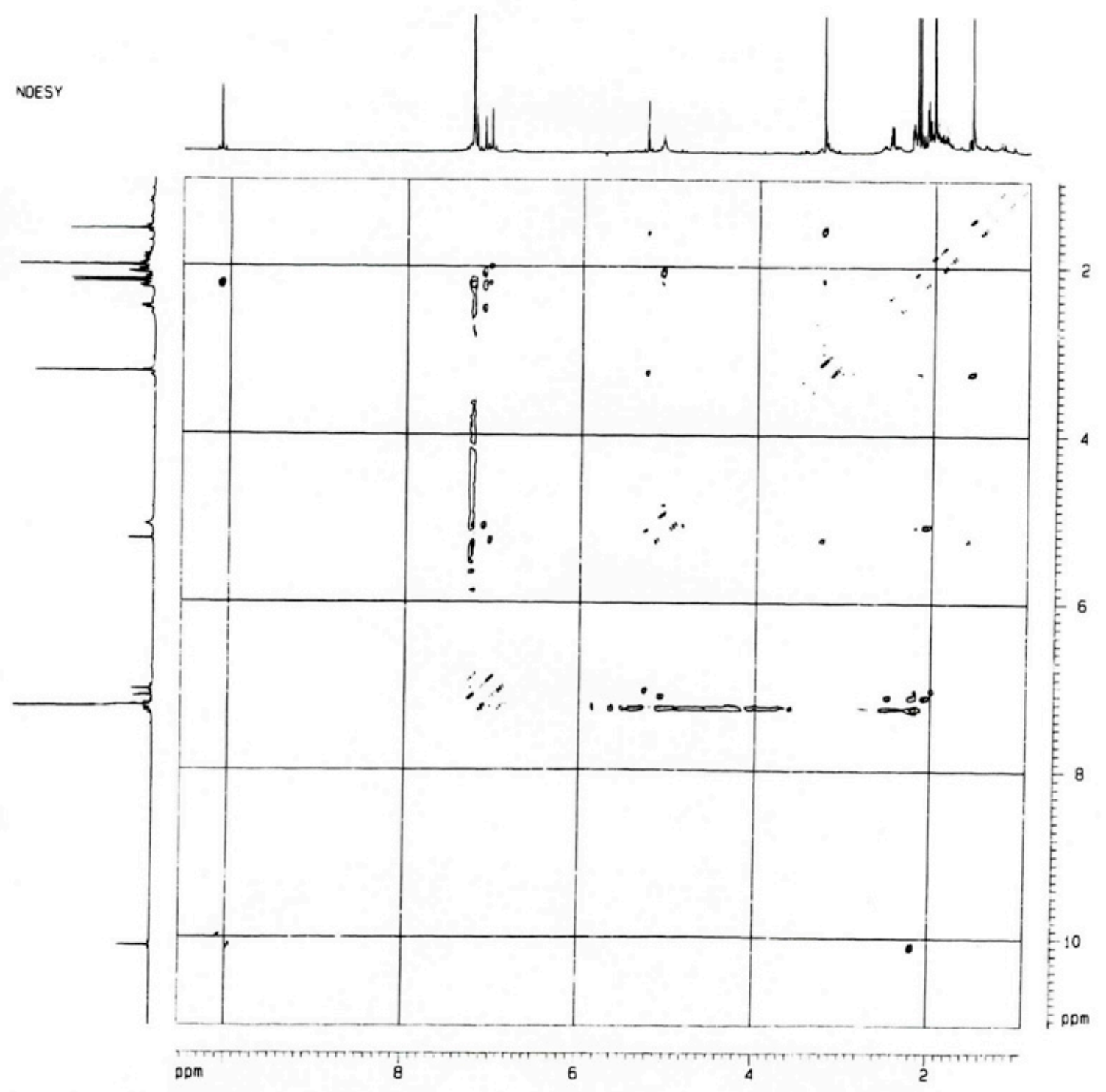

